\title{
Cytokine Storm and Signaling Pathways: Pathogenesis of SARS-CoV-2 Infection, Managing and Treatment Strategies
}

\author{
Rajiv Kumar ${ }^{1 *}$ and Kiran Gulia ${ }^{2}$ \\ ${ }^{1}$ NIET, National Institute of Medical Science, India \\ ${ }^{2}$ Materials and Manufacturing, School of Engineering, University of Wolverhampton, England \\ *Corresponding author: Rajiv Kumar, NIET, National Institute of Medical Science, India
}

\begin{tabular}{|c|c|}
\hline ARTICLE INFO & ABSTRACT \\
\hline Received: 幽 April 26, 2021 & Coronavirus disease is caused by severe acute respiratory syndrome coronavirus 2 \\
\hline Published: 䋃 May 03, 2021 & \\
\hline $\begin{array}{l}\text { Citation: Rajiv Kumar, Kiran Gulia. Cy- } \\
\text { okine Storm and Signaling Pathways: } \\
\text { Pathogenesis of SARS-CoV-2 Infection, } \\
\text { Managing and Treatment Strategies. Bi- } \\
\text { omed J Sci \& Tech Res 35(3)-2021. BJSTR. } \\
\text { MS.ID.005715. }\end{array}$ & 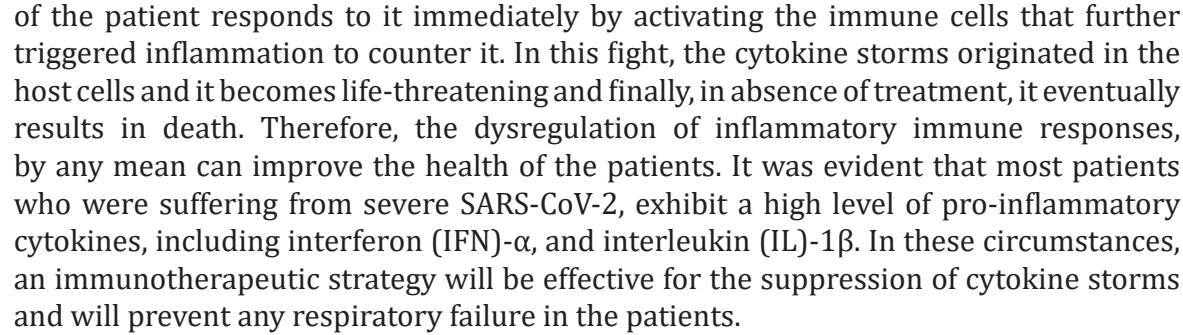 \\
\hline
\end{tabular}

\section{Introduction}

The pathology of cytokine storm is a very complex phenomenon in which a patient's body produces high immune defense to counter external stimuli excessively. The speed of spreading of SARS-CoV-2 infection is too high and therefore, the mortality rate is also high. Understanding the route of SARS-CoV-2 infection can provide deeper insight into outbreak, disease prognosis, and its involvement in the failure of multiple organs including acute respiratory distress syndrome, heart, renal, and liver. All the aforesaid symptoms are life-threatening [1]. The fight between SARS-CoV-2 and immune cells initiated a hyper-inflammatory response in the body that is identified by the pathological cytokine levels. These states are defined as cytokine storm syndrome and observed critical conditions marked the disease severity. It was evident from the observation that ongoing research on SARS-CoV-2 infection expose the causes responsible for the initiation of severe and deteriorated conditions of lungs which are inter-related to the emergence of cytokine storm in patient's bodies. Here, the authors underlined the importance of elucidation of pathogenesis concerned with SARSCoV-2 infection, cytokine storm, and interrelated signaling.

These investigations will propose better treatment strategies for curing SARS-CoV-2 and controlling the induced inflammatory storm. These implemented strategies can suggest better medication guidelines for clinical treatment. The existence of a systemic hyperinflammation confirmed the acute respiratory distress syndrome and finally, these pathological conditions forced a failure in the functioning of many multiple organs [2]. Cytokine storm is the final state that refers to determining the clinical conditions caused by excessive immune reactions and underlined as one of the major causes occurred just because of SARS-CoV-2 infection. Therefore, it identifies a need for better elucidation of the pathogenesis of cytokine storm that has not yet been illuminated [3].

As cytokine storm initiated by SARS-CoV-2 infection, it is also responsible for the origin of various other features of cytokines, 
such as IL-6 and IL-10 [4]. Many clinical trials and evidence have proved that the anti-inflammatory therapy is an effective strategy for a better treatment of SARS-CoV-2 infection. Recently, several remedies are used to treat the cytokine storm labelling it as cytokine-targeted therapies [5]. Varied research efforts are being continued to search a novel an anti-inflammatory treatment that can be useful in the prevention of SARS-CoV-2 infection. Here, the authors provide an overview on cytokine storm and discuss the current approach available for the treatment. They explore various other challenges that existed in cellular components responsible and that initiated the immune response, while dealing with a cytokine storm as well as other biological functions. In the end, the authors again conferred the few potential therapies that were applied to neutralise the cytokine storm.

However, high concentrations of pro-inflammatory cytokines are not the sole reason for the existence of the cytokine, but also there are many other processes that are not yet discovered, and that is why no complete scientific illustration of a cytokine storm possibly exist (Figure 1). Furthermore, there is a need to develop a blueprint of molecular mechanism underlying the cytokine storm that initiates the pathogenesis of SARS-CoV-2 infection [6]. The main cause behind these concepts is to develop more accurate therapeutic strategies that can be applied to prevent a cytokine storm or suppress it at the time of its origin. The aim of author for this short communication, is to define the concept of a cytokine storm, as well its inter-related signaling pathways, concerning biological consequences, and to discover the causes of cytokine overproduction. The authors also tried to explore some other features related to its genomics and tried to reveal more scientific inputs about the kinetics of cytokine and provided more details about the cytokine signaling as well as causes responsible for overlapping of cytokine signaling.

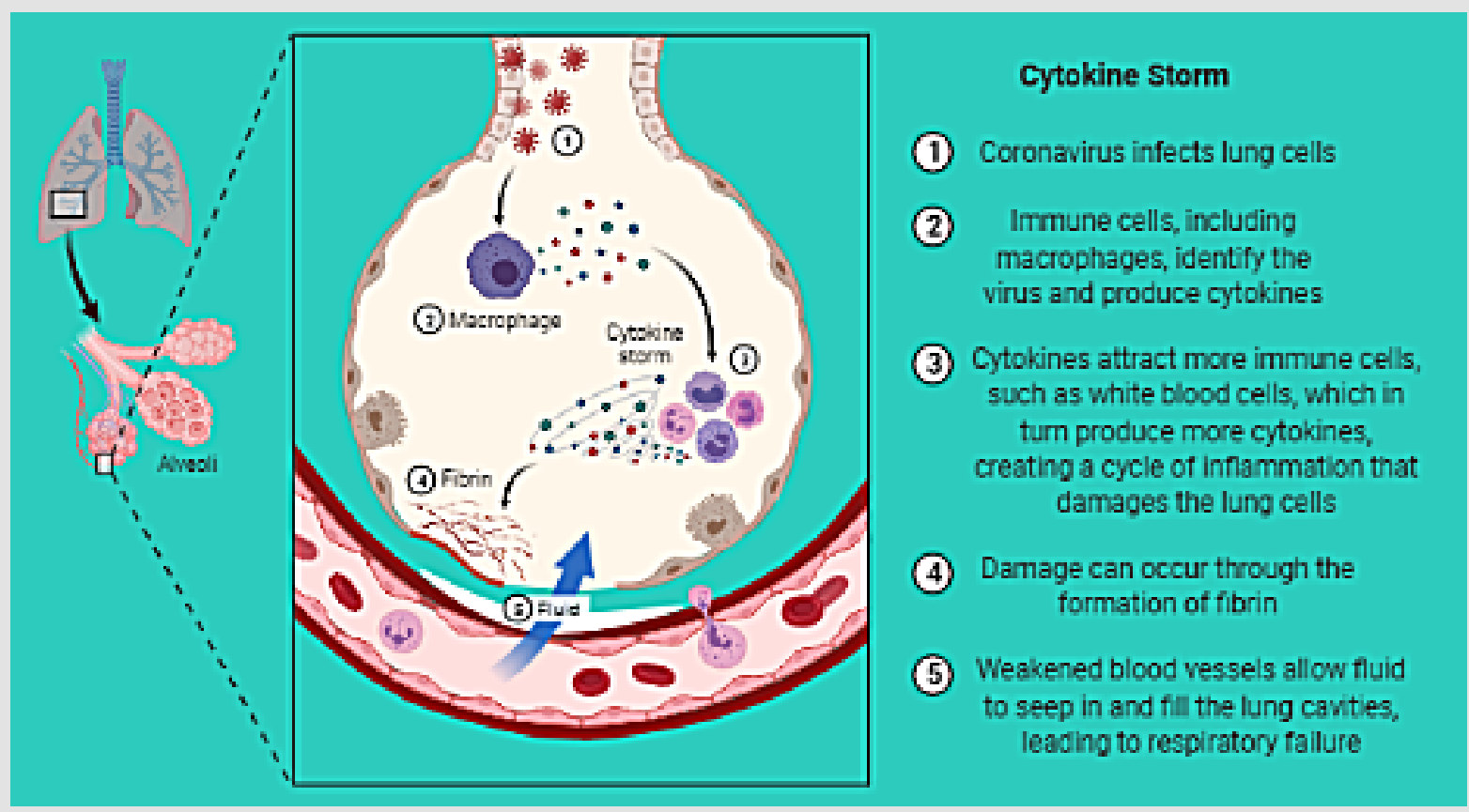

Figure 1: Schematic representation of cytokine storm. "Adapted and created with permission from [biorender.com] and acknowledged.

\section{Background}

At the time of coronavirus attack, over-activation of the immune system transpired, such stages are defined as storm in medical term. A better understanding in the phenomenon of these kind of storms helps to recognise them as cytokine storm. The knowledge of the signaling routes of the storm, and pathogenesis of coronavirus will boost the possibilities to design a successful strategy for managing and planning for developing various treatment strategies [7]. Pathogens (virus or bacteria) must face a strong response of defense from the immune system of the host, during their outbreak and the white blood cells initiate the pinpoint against the pathogens to destroy them. When any pathogen attacked, the immune cells of the host identify it, trigger inflammation and in the meantime, they recruit more immune cells to counter it [8]. Meanwhile, the immune cells release cytokines, and signaling molecules to establish a better communication. Finally, cytokines triggered localized inflammation to destroy the aforesaid pathogens. The immune system keeps producing cytokines, as soon as it is over flooded, that is the sign of the emergence of a cytokine storm.

Later on, the excess inflammation damage multiple organs. Sometimes, an excess amount of cytokine hinders blood supply that affects the resource of oxygen and nutrients supply that is 
transported to the various organs (heart, lungs, and brain). In many cases, a cytokine storm initiates acute respiratory distress syndrome, as a result, the lungs lose their natural functioning, and a hindrance in the supply of oxygen occurs (Figure 2) [9]. In these conditions, sometimes macrophages and white blood cells reach the lungs in a large concentration to kill the infection. It is a fatal situation for a patient. These white blood cells initiate extensive inflammation to counter the pathogens, but sometimes it backfires, and respiratory failure transpires immediately [10].
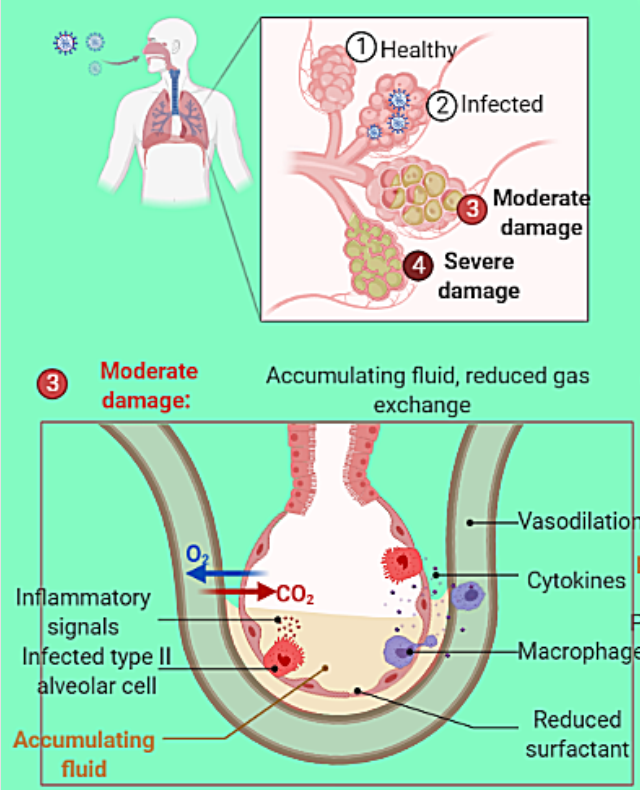

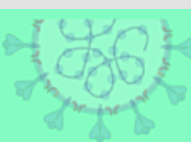

Effects of SARS-CoV-2 on respiration

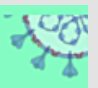

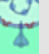

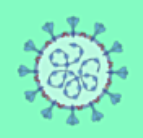

Severe damage: Build up of protein-rich fluid, very limited

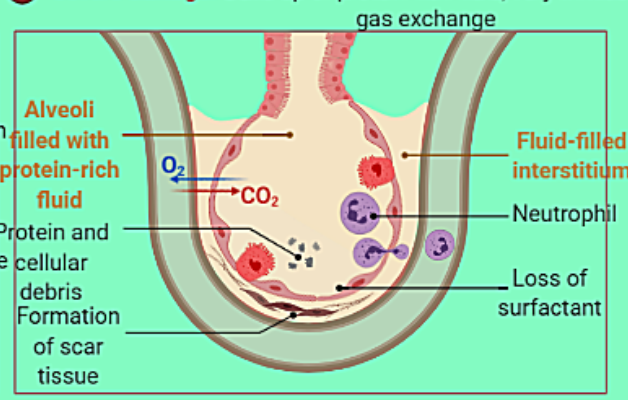

Figure 2: Schematic representation of effects of SARS-CoV-2 on respiration. "Adapted and created with permission from [biorender.com] and acknowledged.

Therefore, special type of remedies are required to calm the cytokine storm, and that are defiantly effective, or, other strategies that can reduce the activation path of immune cells or interference with the route of cytokine production will be helpful. The objective to discuss these strategies is just to propose a remedy that can block the specific signaling pathways by any means and will be fruitful to achieve the goal. All the above-mentioned approaches can be risky because an immunosuppressive intervention, can further initiate the decline in the ratio of important immune cells during SARS-CoV-2 infection. But a remarkable acceleration of clinical trials can address such issues originated at the time of cytokine storm. Simultaneously, there presents an immediate need to search specific strategies to deal with it in most effective scientific manner, because it is a complex physiological and cellular response. There are several other hopes that can be successful in developing more effective remedies for a better treatment of cytokine storm.

\section{Discussion}

To have an objective to develop a treatment and planned management schemes to prevent the pathogenesis of SARS-CoV-2 infection will be an effective idea, but it can be achieved only if, there is a proper understanding of cytokine storms. These strategies can be also useful in the prevention of signaling pathways and in the discovery of hidden aspects of it. Because all these phenomena are interlinked with each other and responsible for the production of proinflammatory cytokines, so there emerges an urgent need to investigate it further. Authors underlined the causes that initiate deterioration in the health of affected patients. It was observed that they are directly linked with dysregulation and excessive release of cytokines. The pathology of infectious disease, as well as the role of cytokine storm, conferred it and how the patients can mount immune responses to deal with the infection originated by SARS$\mathrm{CoV}-2$, is an area that must be investigated [11].

Overall, how immunomodulatory therapies can improve clinical outcomes during severe acute infection, is a topic for further discussion. It was reported earlier that the concentration of proinflammatory cytokines, interleukin (IL)-6, lead to the exhaustion of lymphocytes that are found in high amounts in severely ill patients [12]. Therefore, it was assumed that exploring any possibilities that can explore the mechanisms underlying of host immune responses, will be a pathfinder strategy and it must be discovered to treat the pathogenesis of SARS-CoV-2 infection. Any diagnostic tool that can detect antibodies, will also be helpful for sensitive and accurate detection. Recently, it was observed that IL-6 inhibitor displayed potential as an effective remedy, and it is also a safer one. Besides, 
other remedies including antimalarial agents, corticosteroids, checkpoint inhibition, and intravenous immunoglobulin are useful for counting the cytokine storm [13].

The delivery of immunomodulatory therapies can be guided using a biosensor to enhance the impact of anti-inflammatory treatments. Here, an investigation based on bioinformatics theme was suggested for the elucidation of immune responses initiated and triggered by SARS-CoV-2 infection. These sites are located in primary human lung epithelial and later on transformed into human lung alveolar. Serious scientific intervention is required to explore the mechanisms behind these several complications that causes serious complications during SARS-CoV-2 infection [14]. These events further develop a specific cytokine storm which proceeds in the recruitment of an excessive neutrophil, initiate thrombosis, and initiate damages. The pathway of SARS-CoV-2 infection has different clinical features, biological alterations, and various other mediators of pathophysiology. In brief, these varieties of infection enter into respiratory epithelial cells and pulmonary type-II pneumonitis. Then, these pathogens initiate the production of inflammatory cytokines that is associated with IFN- $\lambda$-dependent epithelial defense.

\section{Signaling Pathways: Cytokine Storm}

The initiation of SARS-CoV-2 infection has occurred due to acute respiratory distress syndrome, which in high severity, depends on a cytokine storm [15]. The interleukin-6 (IL-6) amplifier is one of the components of cytokine storm and perform as a hyper-activation machinery during the regulation of nuclear factor kappa B (NF- $\kappa \mathrm{B})$ pathway. The stimulation of these changes proceeds via activation of IL-6-signal transducer. All these variations transpired like a chain reaction (Figure 3). These intermediators further initiate transcription 3 (STAT3) and NF- $\mathrm{B}$ signaling in epithelial and endothelial cells. By targeting IL-6, new therapeutic strategies can be planned to stop the inflammation.

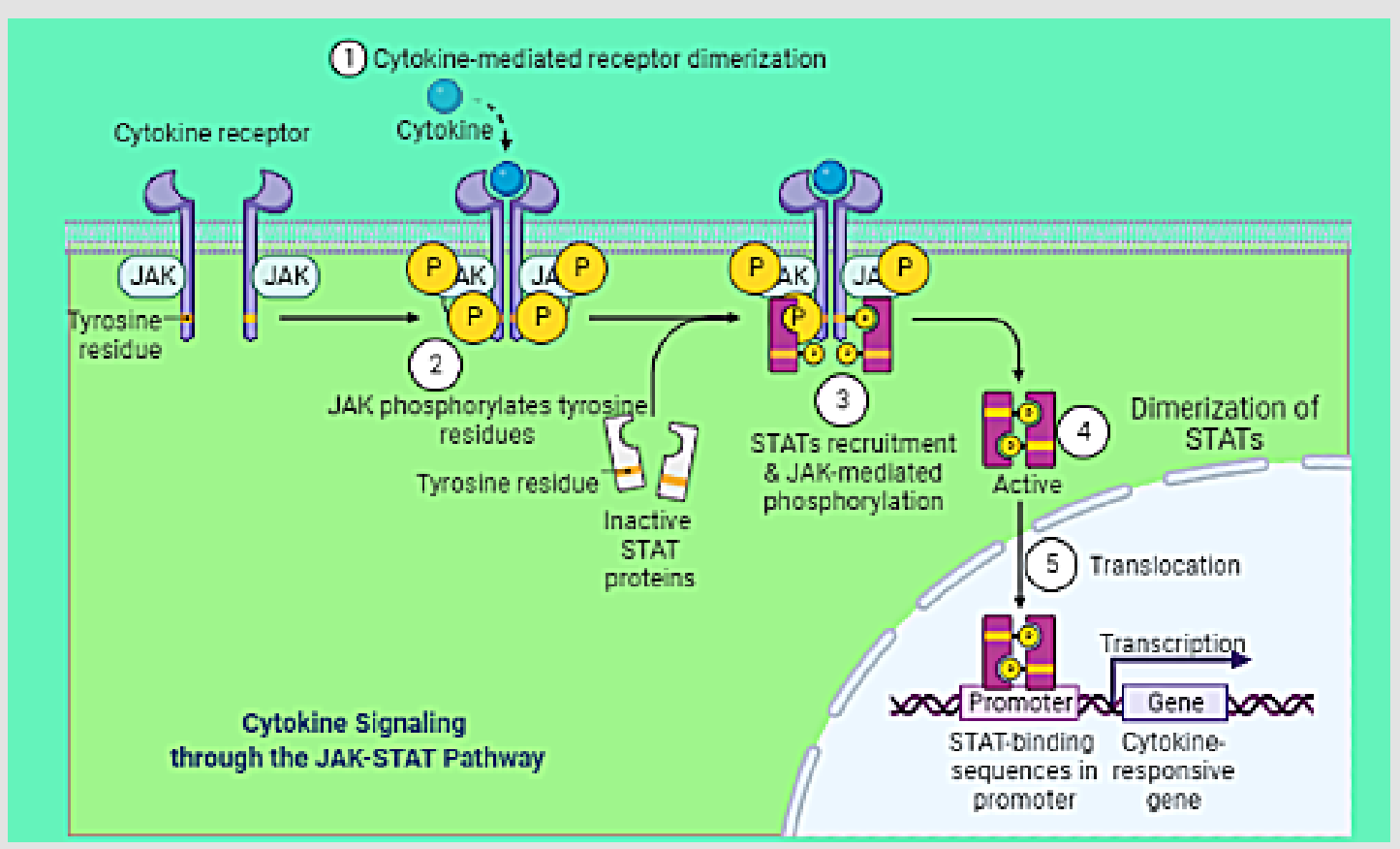

Figure 3: Schematic representation of signaling of cytokine storm. "Adapted and created with permission from [biorender. com] and acknowledged.

By elucidating the signaling pathways of cytokine storm, a new approach can be developed to review the pathogenic mechanism for searching a potential therapeutic target [16]. The pro-inflammatory cytokines such as interleukin (IL)-1B, IL-6, IL-8, IL-17, interferon$\gamma$-inducible protein (IP10), and granulocyte-macrophage colonystimulating factor must be investigated to have a better look on signaling pathways of cytokine release and its conversion into a storm [17].

\section{Conclusion}

Urgent investigation about the physiology of cytokine storm is required for the development preventive and therapeutic drugs. Besides, the immunomodulatory therapies can be a better option for it. Strong immune responses can build a better defence against the viral infection and inflammatory innate response. Overall, it is a well-known knowledge that massive cytokine and chemokine 
dysregulated the host immune defense that are responsible for the formation of a cytokine storm. The option to counter the progression of cytokine storm can only be possible if a proper understanding of immune signaling pathways of the triggered situations when SARSCoV-2 infection attack is available.

These investigations will further reveal the mechanism of molecular cascades participating in various biological processes that intervene with the viral infections and can underline the concerned active molecular events that are responsible for triggering it. A deeper understanding of the mechanism when the immune system initiate dysregulation might be helpful, in the clinical management of SARS-CoV-2 infection and in the designing of prevention strategies. Even though various studies have been reported, once infected by SARS-CoV-2, patients must be considered vulnerable for reappearance of clinical manifestations, especially those that have experienced organ failures and lungs pneumonia pneumonia. It is also of critical importance that once exposed to SARS-CoV-2 infection, patients have rigorously been diagnosed of neurological and psychiatric disorders over following subsequent six months.

\section{Acknowledgement}

One author (Rajiv Kumar) gratefully acknowledges his younger brother Bitto. The author acknowledges bio render to illustrate the online diagram (Figure 1, 2 and 3). The authors acknowledge km. Babita Pundir, Host of FM Rainbow 107.1 MHz, Bombay, India for the kind support and encouragement.

\section{References}

1. Tisoncik JR, Korth MJ, Simmons CP, Farrar J, Martin TR, et al. (2012) Into the Eye of the Cytokine Storm. Microbiol Mol Biol Rev 76(1): 16-32.

2. Fara A, Mitrev Z, Rosalia RA, Assas BM (2020) Cytokine storm and COVID-19: a chronicle of pro-inflammatory cytokines: Cytokine storm: The elements of rage! Open Biology 10(9): 200160.

3. Choudhary S, Sharma K, Silakari O (2021) The interplay between inflammatory pathways and COVID-19: A critical review on pathogenesis and therapeutic options. Microbial Pathogenesis 150: 104673.

4. Altable M, de la Serna JM (2021) Neuroinflammation links COVID-19 and fragile X syndrome: Role of MMP-9, IGF-1, IL-10, metformin, statins and

\section{ISSN: 2574-1241}

DOI: 10.26717/BJSTR.2021.35.005715

Rajiv Kumar. Biomed J Sci \& Tech Res

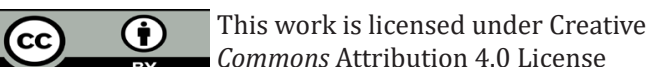

Submission Link: https://biomedres.us/submit-manuscript.php curcumin. Qeios.

5. Wang T, Jiang ZY, Wan XJ, Bian JJ (2020) Cytokine storm and immunoregulatory therapy of coronavirus pneumonia. Acad J Second Mil Med Univ 41(8): 818-823.

6. Jamal M, Bangash HI, Habiba M, Lei Y, Xie T, et al. (2021) Immune dysregulation and system pathology in COVID-19. Virulence 12(1): 918936.

7. Rat $\mathrm{P}$, Olivier E, Dutot M (2020) SARS-CoV-2 vs. SARS-CoV-1 management: Antibiotics and inflammasome modulators potential. Eur Rev Med Pharmacol Sci 24(14): 7880-7885.

8. Kumar R, Chhikara BS, Gulia K, Chhillar M (2021) Cleaning the molecular machinery of cellsviaproteostasis, proteolysis and endocytosis selectively, effectively, and precisely: intracellular self-defense and cellular perturbations. Molecular Omics 17: 11-28.

9. Liu B, Li M, Zhou Z, Guan X. Xiang Y (2020) Can we use interleukin-6 (IL6) blockade for coronavirus disease 2019 (COVID-19)-induced cytokine release syndrome (CRS)? Journal of Autoimmunity 111: 102452.

10. Kumar R, Gulia K (2021) The convergence of nanotechnology-stem cell, nanotopography-mechanobiology, and biotic-abiotic interfaces: Nanoscale tools for tackling the top killer, arteriosclerosis, strokes, and heart attacks. Nano Sel 2(4): 655-687.

11. Teijaro JR, Walsh KB, Rice S, Rosen H, Oldstone MBA (2014) Mapping the innate signaling cascade essential for cytokine storm during influenza virus infection. Proc Natl Acad Sci USA 111(10): 3799-3804

12. Lukan N (2020) “Cytokine storm”, not only in COVID-19 patients. Mini review. Immunology Letters 228: 38-44.

13. Catanzaro M, Fagiani F, Racchi M, Corsini E, Govoni S, et al. (2020) Immune response in COVID-19: addressing a pharmacological challenge by targeting pathways triggered by SARS-CoV-2. Signal Transduction and Targeted Therapy 5(1): 84.

14. Luo W, Li YX, Jiang LJ, Chen Q Wang T, et al. (2020) Targeting JAK-STAT Signaling to Control Cytokine Release Syndrome in COVID-19. Trends in Pharmacological Sciences 41(8): 531-543.

15. Zhou T, Su TT, Mudianto T, Wang J (2020) Immune asynchrony in COVID-19 pathogenesis and potential immunotherapies. Journal of Experimental Medicine 217(10); e20200674.

16. D́elia RV, Harrison K, Oyston PC, Lukaszewski RA, Clark GC (2013) Targeting the 'Cytokine Storm' for Therapeutic Benefit. Clinical and Vaccine Immunology 20(3): 319-327.

17. Crayne CB, Albeituni S, Nichols KE, Cron RQ (2019) The immunology of macrophage activation syndrome. Frontiers in Immunology 10: 119.

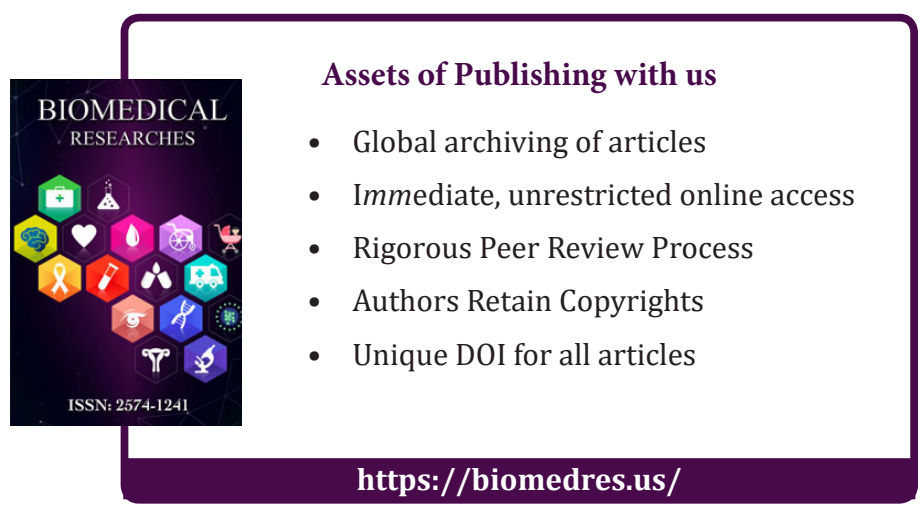

